\title{
RESPONSABILIDAD SOCIAL BANCARIA $Y$ SU INFLUENCIA EN LA COMPETITIVIDAD
}

\section{BANKING SOCIAL RESPONSIBILITY AND ITS INFLUENCE ON COMPETITIVENESS}

\author{
Nataly Amelia Bracamonte Carpio ${ }^{1,}$ \\ https://orcid.org/0000-0001-6625-6883 \\ nataly.bracamonte.carpio@gmail.com \\ Lucio Walter Manuel Valderrama Pérez ${ }^{2}$ \\ Didtps://orcid.org/0000-0001-6061-2736 \\ walter_valderrama@hotmail.com
}

\section{RESUMEN}

A nivel mundial, aumenta la relevancia de la responsabilidad social; así, es fundamental estudiar el contexto de las instituciones bancarias; y determinar de qué manera la responsabilidad social de estas instituciones influye en la competitividad de un Banco con sede en Tacna, año 2019. Se realizó una investigación básica, observacional, transeccional explicativa. La muestra estuvo constituida por los usuarios del Banco, con una muestra total de 384 clientes. El instrumento utilizado fue el cuestionario. Se ha comprobado que la responsabilidad social bancaria influye significativamente en la competitividad del banco. La variable responsabilidad social bancaria tiene un nivel de influencia del $72 \%$ sobre la variable competitividad. ( $\mathrm{R}^{2}$ significativo según el indicador de Nagelkerke).

Palabras claves: responsabilidad social, competitividad, dimensión ética, dimensión social, dimensión ambiental.

\begin{abstract}
At the global level, the relevance of social responsibility is increasing; thus, it is essential to study the context of banking institutions; and determine how the social responsibility of these institutions influences the competitiveness of a Bank based in Tacna, year 2019. A basic, observational, explanatory transectional investigation was carried out. The sample consisted of Bank users, with a total sample of 384 clients. The instrument used was the questionnaire. Banking social responsibility has been found to significantly influence the bank's competitiveness. The bank social responsibility variable has a $72 \%$ level of influence on the competitiveness variable. (R2 significant according to the Nagelkerke indicator).
\end{abstract}

Key words: social responsibility, competitiveness, ethical dimension, social dimension, environmental dimension

\footnotetext{
${ }^{1}$ Universidad Privada de Tacna. Facultad de Ciencias Empresariales. Maestro en Administración y Dirección de Empresas.

2 Universidad Privada de Tacna. Facultad de Derecho y Ciencias Políticas. Doctor en Administración y en Educación..
} 


\section{INTRODUCCIÓN}

En el contexto global, las organizaciones bancarias tratan de elevar la competitividad y rentabilidad, por ello se hace perentorio que desarrollen prácticas de responsabilidad social bancaria, ya que permitirá fortalecer sus beneficios intangibles. La responsabilidad social del sector financiero es una parte importante del éxito de las organizaciones empresariales, pero algunas entidades financieras no la han implementado en su totalidad. La responsabilidad social cooperativa o corporativa (RSC) es una tendencia que se incluye cada vez más en la estrategia organizacional. Para la Dirección de Trabajo del Gobierno de Chile (2005), esta responsabilidad empresarial (RSE) es un estilo de gestión empresarial que reconoce constantemente la relación entre la organización y sus interlocutores. En el Perú, la RSE de la banca debe ser inherente a su actividad empresarial desde los siguientes aspectos: ética, incluyendo compromisos éticos, relaciones transparentes con los grupos de interés y difusión de valores. Por tanto, el trabajo de investigación actual tuvoel objetivo siguiente: Conocer cómo la responsabilidad social influye en la competitividad de los bancos ubicados en Tacna.

De acuerdo al último informe del Anuario de Corresponsables, (2016), da a conocer las prácticas de RSE de diversas organizaciones que valoran sus actividades responsables, y considera que hay evidencia de que las políticas de RSE se van adoptando y fusionando gradualmente optimizar la gestión empresarial. Actualmente, las entidades bancarias implementan diversas estrategias como parte de sus políticas corporativas para participar en el camino de la responsabilidad social empresarial. No obstante, algunas entidades financieras no desarrollan óptimamente prácticas de tan importante aspecto, debido a que no valoran la importancia de desarrollar la responsabilidad social. Uno de los principales bancos en Tacna, por su reputación corporativa, considera políticas de responsabilidad social, y tiene la plena convicción, que si lo desarrollaría en forma efectiva, optimizaría su competitividad, sin embargo, todavía no se concretiza, sobre todo en las dimensiones: ética, grupos de interés, dimensión ambiental y dimensión social.

Es necesario señalar que la industria bancaria puede jugar un papel importante entre el crecimiento económico y la protección del medio ambiente, para promover el desarrollo de instituciones ambientales sostenibles, y jugar un papel a nivel de responsabilidad social, para que sus actividades bancarias puedan ayudar a reducir los problemas globales internos y ambientales, sin embargo, no tienen "tecnología verde" ni proyectos de reducción de la contaminación. El enfoque de la administración de los bancos designados no es reducir los desechos, controlar la contaminación, usar conscientemente los recursos naturales y monitorear preventivamente la administración del ambiente de trabajo desde los empleados hasta los clientes; y según la Guía de ecoeficiencia para empresas del Ministerio de Ambiente del Perú (2009), señala que «la ecoeficiencia se obtiene por medio del suministro de bienes y servicios con precios competitivos, que satisfagan las necesidades humanas y proporcionen calidad de vida, mientras reducen progresivamente los impactos medioambientales y el consumo de recursos a lo largo de su ciclo de vida».

El banco, posee una política medioambiental que tiene las siguientes consideraciones: Esta política es global y se aplica a todas las actividades que realiza el Comité de Ecoeficiencia y Compras Responsables, encargado de coordinar las políticas ambientales y velar por su cumplimiento a través del sistema de gestión ambiental. A pesar de esto, la tecnología LED no se ha adoptado en la sede del banco en Tacna, por lo que es imposible ahorrar electricidad y reducir la generación de energía. En el medio ambiente, esto significa menos contaminación. También desarrolla el reciclaje de papel y cartuchos de tóner, y controla el consumo de papel y agua. Pero no a tiempo. Por otro lado, no participa en proyectos de gestión sostenible, como eficiencia energética, biocombustibles, transporte sostenible y edificios sostenibles, En cuanto a la comunidad, no se evidencia notoriamente que, a través de las inversiones y/o proyectos de inversión, los bancos permitan el mejoramiento de la calidad de vida, impactando a los colaboradores de la organización puesto que son parte de la comunidad, razón por la cual se puede apreciar claramente la cadena de valor. 
En la sede Tacna, el banco no implementa lineamientos ni políticas que promuevan actividades internas que repercutan en la sociedad. En el Ámbito interno no se realizan talleres de responsabilidad social. Sin embargo, como gestión de su capital humano, brinda a los socios igualdad de condiciones y estándares de oportunidad. En cuanto a los colaboradores, no se ha considerado adecuadamente el desarrollo de las personas y familias, sino en materia de salud; seguridad y condiciones laborales; políticas salariales y asistenciales. Pero se observa también que no tienen iniciativas de mejorar el beneficio económico del trabajador durante su permanencía. Con respecto a los grupos de interés orientado a los clientes, si bien es cierto se desarrolla productos y gestión de políticas de marketing y comunicación, ventas éticas, pero no se demuestra a cabalidad excelencia de servicio en atención al consumidor. Por otro lado, en cuanto a los grupos de interés orientado a los proveedores, no se les da un apoyo total para su desarrollo, así como en su evaluación y selección. En cuanto a la comunidad, no existen relaciones fortalecidas con las organizaciones comunitarias de Tacna, no apoyan en forma permanente a proyectos ni a programas sociales en la ciudad. No promueven en gran medida una educación ambiental y compromiso de la organización hacia la comunidad. En cuanto a los grupos de interés del gobierno, no participa en proyectos y liderazgos gubernamentales que afecten a la sociedad. En cuanto a la participación en proyectos gubernamentales, las organizaciones del sector bancario no invertirán permanentemente recursos humanos o técnicos para ejecutar proyectos específicos o localizados. Estos proyectos son ejecutados por gobiernos locales o regionales, o si adoptaron o cumplieron alianzas con entidades públicas, el propósito es mejorar el nivel de desempeño de los funcionarios públicos. Del mismo modo, las entidades bancarias no participan en gran medida en la redacción de recomendaciones sociales y luego en comunicarlas a las autoridades gubernamentales para su aprobación.

Es importante indicar que las entidades del sector bancario no realizan controles ambientales a las empresas o proyectos de sus clientes. La organización no implementa la RSE relacionado a la protección del medio ambiente, en sus estrategias, es decir, que no muestran preocupaciones medioambientales. Es por tales razones que se hizo la siguiente pregunta de investigación ¿De qué manera la responsabilidad social bancaria influye en la competitividad de un Banco de Tacna?. La investigación propuesta busca, mediante la aplicación de la teoría y los conceptos básicos sobre responsabilidad social con respecto a la dimensión ética, social, ambiental y la competitividad, encontrar explicaciones a situaciones internas del Banco. Es de entender que la relación entre competitividad y responsabilidad, son variables importantes debido a que beneficiaría a los grupos de interés de las entidades financieras, ya que al desarrollar un buen aspecto ético, una relación buena con los grupos de interés, un adecuado aspecto ambiental y aspecto social, que conllevaría a que los entes financieros al diferenciarse de los demás, sean más competitivos que otras organizaciones financieras, lo que conducirá a elevar su cartera de clientes y, por ende, su rentabilidad.

Cea (2010) explica que las acciones de responsabilidad social corporativa implica el desarrollo de acciones éticas y altruistas. Es decir, muestran interés social hacia la comunidad, a los grupos de interés y determinó que las entidades bancarias deben priorizar los grupos de interés, que contribuyen a que eleve su buen desarrollo de responsabilidad social empresarial, su imagen corporativa y su rentabilidad. Rosero (2015) demuestra que la empresa responsable socialmente, se basa en los impactos sociales y medioambientales que se ocasionan cuando desarrollan sus tareas y actividades. Asimismo, se demostró que existe una relación positiva de la RSE sobre el desempeño financiero. Los bancos consideraron el componente social, medioambiental y de gobierno corporativo, lo que ha contribuido a elevar su imagen empresarial. Wong (2015) describe que antes de organizar la responsabilidad de la entidad, es necesario maximizar las ganancias para sus accionistas y clientes. El propósito es establecer una cultura financiera especulativa, brindar credibilidad a los prestatarios y excluir financieramente a los grupos sociales desfavorecidos; financiar la responsabilidad social que la entidad puede asumir asegurará que el negocio bancario tenga un sentido de responsabilidad social para establecer una cultura financiera; la sostenibilidad permite optimizar la calidad de vida de los grupos de interés en los aspectos económicos, sociales y ambientales. Truño (2016) Dijo que se puede constatar que la imagen de la entidad está relacionada con la satisfacción del consumidor. Por tanto, es necesario destacar que las entidades bancarias deben dar prioridad a sus grupos de interés. En los 
siguientes aspectos, la responsabilidad social del banco debe ser inherente a sus actividades comerciales: ética, incluidos sus compromisos, relaciones transparentes con los grupos de interés y divulgación de valores. En cuanto a los colaboradores, considera el respeto a las personas y la diversidad, el desarrollo profesional y la empleabilidad, la salud, la seguridad y las condiciones laborales. Y responsabilidad social en el aspecto ambiental, incluido el compromiso de mejorar la calidad ambiental, mejorar los procesos de gestión ambiental y contribuir a la protección del medio ambiente.

Para Bocanegra (2010) lamentablemente en Perú, todavía la responsabilidad social empresarial no tiene fuerza, uno de los aspectos cruciales es que cumplan con la legislación vigente en el rubro de los entes financieros, es decir, teniendo en cuenta el principio de legalidad; confirma que la comunicación de responsabilidad social corporativa del Banco de la Nación en el programa de responsabilidad empresarial contribuye a que la institución consiga sus objetivos institucionales. En cambio para la visión y componentes estratégicos de Corredor et al. (2016) son considerados como un alto foco de la industria bancaria de Colombia. En la industria bancaria de Colombia, se comprenden las necesidades de los clientes, la implementación de estrategias y metas está relacionada con la responsabilidad social corporativa y sus diferentes beneficios, el grupo está alineado, aumenta el compromiso con la sostenibilidad y la durabilidad a través de su modelo de negocio, y la sección de gobierno corporativo y gestión destaca el cumplimiento de la industria con las prácticas de gobierno corporativo, sostenibilidad y anticorrupción. Por ello, las entidades bancarias cumplen a cabalidad con la normativa para no generar negocio o dinero por actividades ilícitas.

Para ello, desde la perspectiva de la responsabilidad social corporativa, para Canto et al. (2016), la industria bancaria peruana tiene un mayor grado de integración con los stakeholders "clientes", enfocándose en sus procesos, políticas y estrategias para lograr el respeto a la privacidad del consumidor, excelente cliente servicio y ventas éticas. En cuanto a los "accionistas" y "proveedores" de los grupos de interés, la percepción de los bancos socios peruanos es que el departamento toma acciones para establecer relaciones con la competencia, desarrollar principios éticos y está comprometido con el buen gobierno corporativo. Por el lado de los proveedores, existe un estricto y transparente método para evaluarlos y seleccionarlos. Confirmaron que al establecer buenas relaciones con los grupos de interés, Hidalgo (2018) confirmó que la responsabilidad social empresarial afecta la imagen institucional percibida por los clientes y el equilibrio social afecta la imagen institucional percibida por los clientes. De igual forma, Velarde (2019) afirmó que la responsabilidad social corporativa tiene un impacto significativo en el posicionamiento de las entidades de Tacna Bank; para la variable "ubicación", se realiza de la misma manera en cada dimensión de la variable "responsabilidad social corporativa". Asimismo, se encuentra que la dimensión social tiene un impacto significativo en el posicionamiento de las entidades de Tacna.

\section{METOdOLOGÍA}

La investigación básica, pues procura la acumulación del conocimiento que se va profundizando conforme se avanza en el descubrimiento de nuevos fenómenos, nuevos hechos, nuevas teorías, entre otras cosas (Sánchez, 2019). El presente estudio es de diseño no experimental, transeccional. El ámbito de acción de la presente investigación está circunscrita en toda la ciudad de Tacna (Banco Continental sede Tacna). El tiempo social del presente trabajo de investigación se encuentra comprendido en el año 2019. Las unidades de análisis son los usuarios de un banco local.

En el presente estudio estuvo constituida por los usuarios de un banco local; por lo que se considera una población desconocida, que tiene un tratamiento especial para la determinación de la muestra. Para el presente estudio se determinó la muestra 384 usuarios, con nivel de confianza utilizado (95\%). Con el propósito de generalizar los resultados a la población de usuarios, se seleccionó de acuerdo con el criterio de la varianza máxima para un muestreo probabilístico y para una población infinita, la cual arrojo un tamaño de 384 usuarios. La Responsabilidad social bancaria, fue medida por sus dimensiones: 
Dimensión 1: Dimensión ética (compromisos éticos, relaciones transparentes con las partes interesadas, divulgación de valores)

Dimensión 2: Grupos de interés ( Responsabilidad social con los colaboradores, responsabilidad social con los clientes, Responsabilidad social con los órganos reguladores y gobierno)

Dimensión 3: Dimensión ambiental ( Compromiso con la mejora de la calidad ambiental, Mejora de procesos de gestión ambiental, Contribución a la conservación del medio ambiente)

Dimensión 4: Dimensión social (Organización de la acción social de la empresa, Participación en las actividades sociales, Informe de acciones sociales, Balance social)

La Competitividad, fue medida por sus dimensiones:

Dimensión 1: Factores internos de la empresa (Capacidad tecnológica, calidad de los recursos humanos, Conocimiento de mercado y adaptabilidad)

Dimensión 2: Factores sistémicos (Infraestructura tecnológica e institucional)

Dimensión 3: Factores de desarrollo microeconómico (Capacitación y gestión tecnológica de la empresa, nuevos esquemas organizacionales, estrategias de ventas y relaciones con abastecedores)

La recolección de la información se desarrolló en forma personal y de primera fuente, para tal efecto se coordinó con los directivos del Banco. Antes de aplicar los referidos instrumentos, se hizo conocer a los trabajadores de la indicada institución, el tema de la investigación, objetivo del estudio , utilidad de la investigación, Indicaciones sobre el llenado de los instrumentos, el tiempo que sea para el llenado, recomendaciones para el respectivo llenado, se procedió a la entrega de los instrumentos, dándoles un tiempo prudente de una hora cronológica para cada instrumento de acuerdo a las variables de estudio, culminado el llenado de los instrumentos por los usuarios. Los instrumentos cumplieron dos requisitos: Validez y confiabilidad. Se desarrolló la validez de contenido de los instrumentos a través de criterio de expertos, con relación a los instrumentos: Responsabilidad social bancaria y competitividad. Para la confiabilidad se aplicó el modelo estadístico Alfa cronbach. El valor obtenido fue de 0.961 . para "Responsabilidad social bancaria" y 0.941 para el instrumento aplicado a la variable "Competitividad".

\section{RESULTADOS}

Según los usuarios del Banco, el 36,5\% refieren que la responsabilidad social bancaria se encuentra en un nivel bajo. Asimismo, el $55,4 \%$ de los usuarios encuestados manifestaron que se encuentra en un nivel regular y un $8,1 \%$ indicaron que se encuentra en un nivel alto. El $40,4 \%$ refieren que la competitividad se encuentra en un nivel bajo, asimismo, el $49 \%$ de los usuarios encuestados manifestaron que se encuentra en un nivel regular y un $10,7 \%$ indicaron que se encuentra en un nivel alto.

Tabla 1

Nivel de responsabilidad social y competitividad

\begin{tabular}{llrrr}
\hline & & $\mathrm{N}$ & $\%$ & $\%$ acumulado \\
\hline \multirow{3}{*}{ Responsabilidad social } & Bajo & 140 & 36,5 & 36,5 \\
bancaria & Regular & 213 & 55,4 & 91,9 \\
& Alto & 31 & 8,1 & 100,0 \\
& Total & 384 & 100,0 & \\
\hline \multirow{3}{*}{ Competitividad } & Bajo & 155 & 40,4 & 40,4 \\
& Regular & 188 & 49,0 & 89,3 \\
& Alto & 41 & 10,7 & 100,0 \\
& Total & 384 & 100,0 & \\
\hline
\end{tabular}


El 44,3\% refieren que la dimensión ética se encuentra en un nivel bajo. Asimismo, el $45,1 \%$ de los usuarios encuestados manifestaron que se encuentra en un nivel regular, el 50,5\% de los usuarios encuestados manifestaron que la dimensión grupos de interés se encuentra en un nivel regular. El $68,2 \%$ de los usuarios encuestados manifestaron que la dimensión ambiental se encuentra en un nivel regular. Asimismo, el $68,8 \%$ de los usuarios encuestados manifestaron que la dimensión social se encuentra en un nivel regular.

Tabla 1

Dimensiones de la responsabilidad social

\begin{tabular}{llrrr}
\hline $\begin{array}{llrl}\text { Dimensiones de la } \\
\text { responsabilidad social }\end{array}$ & $\mathrm{N}$ & $\%$ & $\%$ acumulado \\
\hline Dimensión ética & Bajo & 170 & 44,3 & 44,3 \\
& Regular & 173 & 45,1 & 89,3 \\
& Alto & 41 & 10,7 & 100,0 \\
& Total & 384 & 100,0 & \\
\hline Dimensión grupos & Bajo & 138 & 35,9 & 35,9 \\
de interés & Regular & 194 & 50,5 & 86,5 \\
& Alto & 52 & 13,5 & 100,0 \\
& Total & 384 & 100,0 & \\
\hline Dimensión & Bajo & 91 & 23,7 & 23,7 \\
ambiental & Regular & 262 & 68,2 & 91,9 \\
& Alto & 31 & 8,1 & 100,0 \\
& Total & 384 & 100,0 & \\
\hline Dimensión social & Bajo & 80 & 20,8 & 20,8 \\
& Regular & 264 & 68,8 & 100,0 \\
& Alto & 40 & 10,4 & \\
\hline
\end{tabular}

Dimensión Ética: Según los usuarios del Banco los compromisos éticos se encuentran en un nivel regular (el 53,9\%). El 43,5\% refieren que las relaciones transparentes con las partes interesadas se encuentran en un nivel bajo. Asimismo, el $44,3 \%$ de los usuarios encuestados manifestaron se encuentran en un nivel regular. Asimismo, el $59,4 \%$ de los usuarios encuestados manifestaron que la divulgación de valores que se encuentra en un nivel regular.

Dimensión Grupos De Interés: Según los usuarios del Banco, en la responsabilidad social con los colaboradores el $69,5 \%$ de los usuarios encuestados manifestaron que se encuentra en un nivel regular. El 55,5\% de los usuarios encuestados manifestaron que la responsabilidad social con los clientes se encuentra en un nivel regular. El $46,4 \%$ refieren que la responsabilidad social con los órganos reguladores y gobierno se encuentra en un nivel bajo.

Dimensión Ambiental: Según los usuarios del Banco el compromiso con la mejora de la calidad ambiental se encuentra en un nivel regular (67,2\%). Que la mejora de procesos de gestión ambiental se encuentra en un nivel regular $(55,7 \%)$. Que la contribución a la conservación del medio ambiente se encuentra en un nivel regular $(63,5 \%)$.

Dimensión Social: El 51,3\% de los usuarios encuestados manifestaron que la organización acción social de la empresa se encuentra en un nivel regular. Que la participación en las actividades sociales en el $58,3 \%$ de los usuarios se encuentra en un nivel regular. De acuerdo al informe de acciones sociales se encuentra en un $58,1 \%$ en un nivel regular. $Y$ que la responsabilidad social en su balance social se encuentra en el $50,8 \%$ de los usuarios encuestados en un nivel regular. 
Tabla 3

Indicadores por dimensión de la responsabilidad social

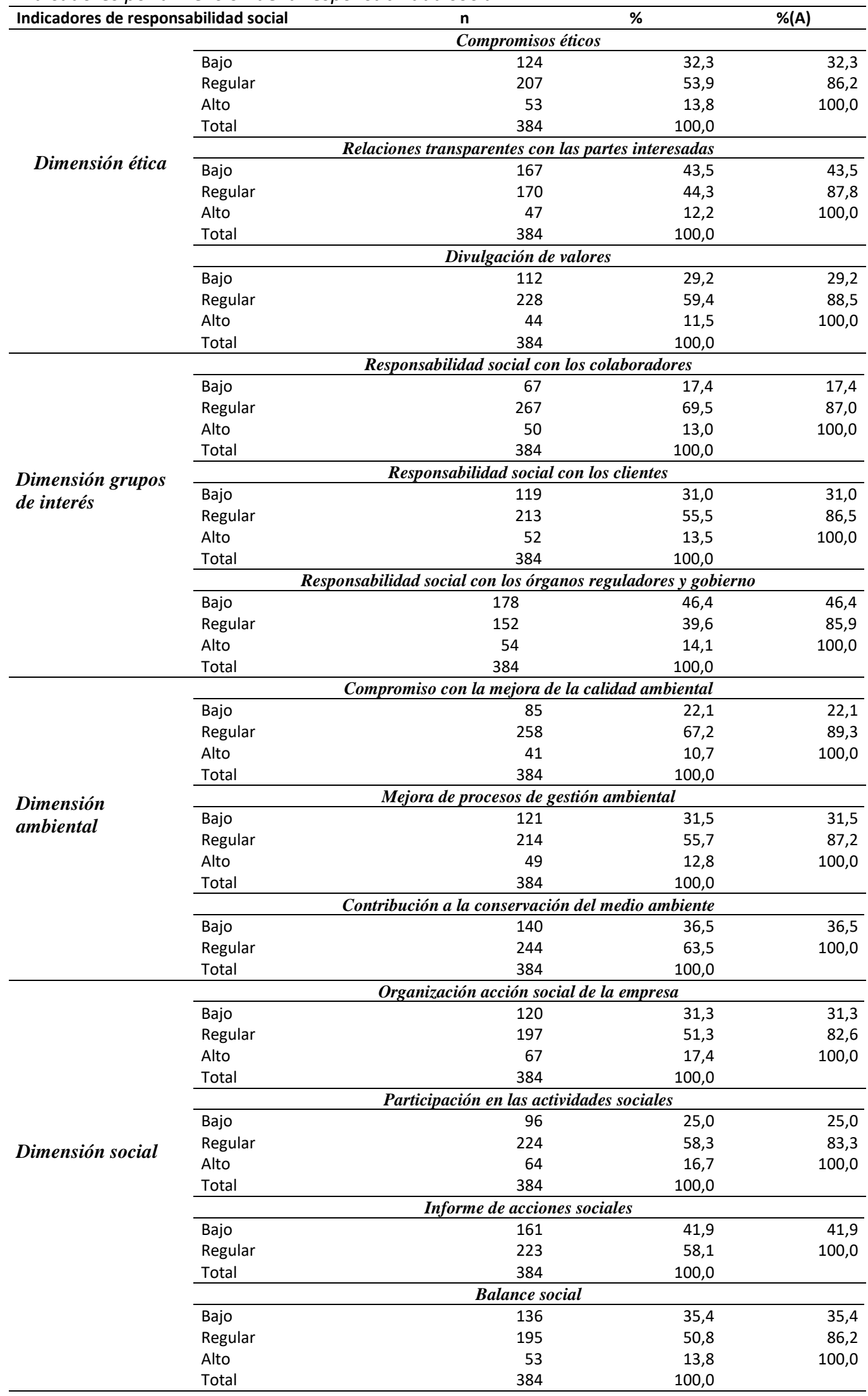


Según los usuarios del Banco, el 51\% refieren que la competitividad en los factores internos se presenta en un nivel bajo. Asimismo, el $36,5 \%$ de los usuarios encuestados manifestaron que se presenta en un nivel regular. El $41,7 \%$ refieren que la competitividad en su capacidad tecnológica se encuentra en un nivel bajo. Asimismo, el $44 \%$ de los usuarios encuestados manifestaron que se encuentra en un nivel regular. El 31,3\% refieren que la competitividad en la calidad de los recursos humanos se encuentra en un nivel bajo. Asimismo, el 58,3\% de los usuarios encuestados manifestaron que se encuentra en un nivel regular y un $10,4 \%$ indicaron que se encuentra en un nivel alto. El $45,8 \%$ refieren que la competitividad en cuanto al conocimiento de mercado y adaptabilidad se encuentra en un nivel bajo. Asimismo, el $40,1 \%$ de los usuarios encuestados manifestaron que se encuentra en un nivel regular y un $14,1 \%$ indicaron que se encuentra en un nivel alto.

Tabla 4

Dimensiones del a competitividad

\begin{tabular}{llrrr}
\hline \multicolumn{2}{l}{ Dimensiones de la competitividad } & Frecuencia & Porcentaje & $\begin{array}{r}\text { Porcentaje } \\
\text { acumulado }\end{array}$ \\
\hline Factores internos de & Bajo & 196 & 51,0 & 51,0 \\
la empresa & Regular & 140 & 36,5 & 87,5 \\
& Alto & 48 & 12,5 & 100,0 \\
& Total & 384 & 100,0 & \\
\hline Factores sistémicos & Bajo & 85 & 22,1 & 22,1 \\
& Regular & 258 & 67,2 & 89,3 \\
& Alto & 41 & 10,7 & 100,0 \\
& Total & 384 & 100,0 & \\
\hline Factores de & Bajo & 118 & 30,7 & 30,7 \\
desarrollo & Regular & 230 & 59,9 & 90,6 \\
microeconomico & Alto & 36 & 9,4 & 100,0 \\
& Total & 384 & 100,0 & \\
\hline
\end{tabular}

\section{DIMENSIÓN: FACTORES SISTÉMICOS}

Según los usuarios del Banco Continental sede Tacna, el 22,1\% refieren que la competitividad en cuanto a sus factores sistémicos se presenta en un nivel bajo. Asimismo, el 67,2\% de los usuarios encuestados manifestaron que se presenta en un nivel regular y un $10,7 \%$ indicaron que se presenta en un nivel alto.

Según los usuarios del Banco Continental sede Tacna, el 22,1\% refieren que la competitividad en cuanto a su infraestructura tecnológica e institucional se encuentra en un nivel bajo. Asimismo, el $67,2 \%$ de los usuarios encuestados manifestaron que se encuentra en un nivel regular y un $10,7 \%$ indicaron que se encuentra en un nivel alto.

\section{DIMENSIÓN: FACTORES DE DESARROLLO MICROECONÓMICO}

Según los usuarios del Banco Continental sede Tacna, el 30,7\% refieren que la competitividad en cuanto a sus factores de desarrollo microeconómico se presenta en un nivel bajo. Asimismo, el 59,9\% de los usuarios encuestados manifestaron que se presenta en un nivel regular y un $9,4 \%$ indicaron que se encuentra en un nivel alto.

Según los usuarios del Banco Continental sede Tacna, el 26,6\% refieren que la competitividad en cuanto a su capacitación y gestión tecnológica del banco se encuentra en un nivel bajo. Asimismo, el $62,5 \%$ de los usuarios encuestados manifestaron que se encuentra regular y un $10,9 \%$ indicaron que se encuentra en un nivel alto.

Según los usuarios del Banco Continental sede Tacna, el 42,7\% refieren que la competitividad en cuanto a los nuevos esquemas organizacionales se encuentra en un nivel bajo. Asimismo, el $43 \%$ de 
los usuarios encuestados manifestaron que se encuentra en un nivel regular y un $14,3 \%$ indicaron que se encuentra en un nivel alto.

Según los usuarios del Banco Continental sede Tacna, el 18\% refieren que la competitividad en cuanto a las estrategias de ventas de servicios se encuentra en un nivel bajo. Asimismo, el $72,7 \%$ de los usuarios encuestados manifestaron que se encuentra en un nivel regular y un $9,4 \%$ indicaron que se encuentra en un nivel alto.

Según los usuarios del Banco Continental sede Tacna, el 30,7\% refieren que la competitividad en cuanto a sus recursos humanos se encuentra en un nivel bajo. Asimismo, el $54,4 \%$ de los usuarios encuestados manifestaron que se encuentra en un nivel regular y un $14,8 \%$ indicaron que se encuentra en un alto nivel.

\section{Tabla 5}

Indicadores de la competitividad

\begin{tabular}{|c|c|c|c|c|}
\hline Indicadores de Competitividad & & $\mathrm{n}$ & $\%$ & $\%(A)$ \\
\hline \multirow{15}{*}{$\begin{array}{l}\text { Factores internos de la } \\
\text { empresa }\end{array}$} & \multicolumn{4}{|c|}{ Capacidad tecnológica } \\
\hline & Bajo & 160 & 41,7 & 41,7 \\
\hline & Regular & 169 & 44,0 & 85,7 \\
\hline & Alto & 55 & 14,3 & 100,0 \\
\hline & Total & 384 & 100,0 & \\
\hline & \multicolumn{4}{|c|}{ Calidad de los recursos humanos } \\
\hline & Bajo & 120 & 31,3 & 31,3 \\
\hline & Regular & 224 & 58,3 & 89,6 \\
\hline & Alto & 40 & 10,4 & 100,0 \\
\hline & Total & 384 & 100,0 & \\
\hline & \multicolumn{4}{|c|}{ Conocimiento de mercado y adaptabilidad } \\
\hline & Bajo & 176 & 45,8 & 45,8 \\
\hline & Regular & 154 & 40,1 & 85,9 \\
\hline & Alto & 54 & 14,1 & 100,0 \\
\hline & Total & 384 & 100,0 & \\
\hline \multirow{5}{*}{ Factores sistémicos } & \multicolumn{4}{|c|}{ Infraestructura tecnológica e institucional } \\
\hline & Bajo & 85 & 22,1 & 22,1 \\
\hline & Regular & 258 & 67,2 & 89,3 \\
\hline & Alto & 41 & 10,7 & 100,0 \\
\hline & Total & 384 & 100,0 & \\
\hline \multirow{20}{*}{$\begin{array}{l}\text { Factores de desarrollo } \\
\text { microeconomico }\end{array}$} & \multicolumn{4}{|c|}{ Capacitación y gestión tecnológica del banco } \\
\hline & Bajo & 102 & 26,6 & 26,6 \\
\hline & Regular & 240 & 62,5 & 89,1 \\
\hline & Alto & 42 & 10,9 & 100,0 \\
\hline & Total & 384 & 100,0 & \\
\hline & \multicolumn{4}{|c|}{ Nuevos esquemas organizacionales } \\
\hline & Bajo & 164 & 42,7 & 42,7 \\
\hline & Regular & 165 & 43,0 & 85,7 \\
\hline & Alto & 55 & 14,3 & 100,0 \\
\hline & Total & 384 & 100,0 & \\
\hline & \multicolumn{4}{|c|}{ Estrategias de ventas de servicios } \\
\hline & Bajo & 69 & 18,0 & 18,0 \\
\hline & Regular & 279 & 72,7 & 90,6 \\
\hline & Alto & 36 & 9,4 & 100,0 \\
\hline & Total & 384 & 100,0 & \\
\hline & \multicolumn{4}{|c|}{ Recursos humanos } \\
\hline & Bajo & 118 & 30,7 & 30,7 \\
\hline & Regular & 209 & 54,4 & 85,2 \\
\hline & Alto & 57 & 14,8 & 100,0 \\
\hline & Total & 384 & 100,0 & \\
\hline
\end{tabular}

Los resultados indican que se estaría explicando la influencia de la responsabilidad social en la competitividad; asimismo se tiene al valor chi cuadrado de 364,722 y p valor : 0,000. La prueba del 
pseudo R cuadrado, según el indicador de Nagelkerke, nos explica que la variable responsabilidad social bancaria tiene un nivel de influencia del $72 \%$ sobre la variable competitividad.

\section{DISCUSIÓN}

De acuerdo con los resultados, se ha confirmado que la responsabilidad social bancaria tiene un impacto significativo en la competitividad de la sede de Tacna en 2019, la prueba R-cuadrada realizada según el indicador de Nagelkerke muestra que las variables de responsabilidad social bancaria tienen un impacto, sobre las variables de competitividad en un $72 \%$. Los hallazgos encontrados se relacionan en parte con la conclusión establecida por Cea (2010), que concluyó que el comportamiento de responsabilidad social empresarial implica el desarrollo de un comportamiento ético y altruista. En otras palabras, mostró interés social en comunidades y grupos de interés. EI resultado hipotético determina que la entidad bancaria de prioridad a los grupos de interés, los hallazgos encontrados se parecen a lo informado por Rosero (2015), quien concluye que la empresa responsable socialmente, se basa en los impactos sociales y medioambientales que se ocasionan cuando desarrollan sus tareas y actividades. Las entidades bancarias implementan las políticas de responsabilidad social que son publicados en la Comisión Europea. Asimismo, se demostró que existe una relación positiva de la RSE sobre el desempeño financiero. Los bancos consideraron el componente social, medioambiental y de gobierno corporativo, lo que ha contribuido a elevar su imagen empresarial.

De igual forma, se ha determinado que en 2019, la responsabilidad social bancaria año 2019, se desarrolla de forma poco efectiva en un 55,4 \% y nada efectiva en un 36,5\%. Estos hallazgos están relacionados en parte con la afirmación de Wong (2015). Wong afirmó que antes de que la entidad organizacional asuma la responsabilidad, debe maximizar las ganancias para sus accionistas y clientes. El propósito es mejorar una cultura financiera especulativa, prestatarios solventes y hacer una sociedad desfavorecida de exclusión económica. La responsabilidad social que pueda asumir las entidades financieras garantizará que el negocio bancario tenga un compromiso social para crear una cultura financiera que posibilite el uso ético y sostenible de la moneda. Se identifican dos dimensiones que delimitan la profundidad del compromiso social de la Institución Financiera: La promoción del ahorro e inversión socialmente responsable, son dimensiones que hacen notar el compromiso social del ente financiero.

Además, se ha comprobado que la responsabilidad social se relacionan de manera directa con la aplicación en los créditos bancarios - Ecuador. Los resultados encontrados se parecen en parte con lo informado por Truño (2016), quien concluyó que la imagen de la entidad se relaciona con la satisfacción de los consumidores; por lo que se hace énfasis que los entes bancarios deben dar prioridad a sus grupos de interés como los clientes, proveedores, y sobre todo la comunidad. La responsabilidad social bancaria debe ser inherente a sus actividades empresariales, en cuanto a las dimensiones: ética, que incluye los compromisos éticos, las relaciones transparentes con las partes interesadas y la divulgación de valores. En lo referente a la dimensión colaboradores, considera el respeto al individuo y diversidad, el desarrollo profesional y la empleabilidad, el cuidado de la salud, seguridad y condiciones de trabajo. Así como la responsabilidad social en la dimensión ambiental, que incluye el compromiso con la mejoría de la calidad ambiental, la mejora de procesos de gestión ambiental y la contribución a la conservación del medio ambiente.

Los resultados encontrados se relacionan en parte con lo informado por Bocanegra (2010), quien concluye que, lamentablemente en Perú, todavía la responsabilidad social empresarial no tiene fuerza, uno de los aspectos cruciales es que cumplan con la legislación vigente en el rubro de los entes financieros, es decir, teniendo en cuenta el principio de legalidad. Es necesario que haya más empresas que desarrollen buenas prácticas de responsabilidad social empresarial a nivel nacional, con el objetivo de elevar el desarrollo humano sostenible. De acuerdo a la hipótesis se confirmó que la comunicación de responsabilidad social corporativa del Banco de la Nación en el programa de responsabilidad empresarial contribuye a que la institución consiga sus objetivos institucionales. 
Se ha demostrado que el nivel de competitividad en el Banco Continental, sede Tacna, año 2019 es regular en un 49 \% y 40 \% en un nivel bajo. Los hallazgos están relacionados en parte con la afirmación de Corredor et al. (2016), quienes concluyeron que el indicador Ethos-IARSE alcanzó un 95\% de cumplimiento en los componentes de visión y estrategia, lo que indica que la industria está muy preocupada por comprender los requisitos de la banca colombiana, y trabajar con diferentes grupos de interés para implementar estrategias y metas consistentes con la responsabilidad social empresarial, aumentar así su compromiso con la sustentabilidad a través de su modelo de negocio. En gobierno corporativo y administración, el indicador Ethos-IARSE logró un $89 \%$ de cumplimiento corporativo, destacando el cumplimiento de la industria con las prácticas de gobierno, sostenibilidad y anticorrupción. Por lo tanto, las entidades bancarias cumplen cabalmente con la normativa para evitar la obtención de negocios o dinero a través de actividades ilícitas; para ello, la gestión y el compromiso pueden verse reflejados en sus informes financieros y mediante la implementación y desarrollo de planes de responsabilidad social corporativa. Asimismo, los hallazgos son similares a los de Beltrán, Quintana e Hidalgo (2016), quienes concluyeron que si bien Scotiabank ha implementado algunas iniciativas dentro de la organización para promover la igualdad de género, no lo hicieron. Es parte de una estrategia de diversificación a largo plazo. No implementó dos recomendaciones: primero, establecer metas claras para la gestión de la mujer en toda la organización, lo que llevó a un enfoque planificado; y segundo, los resultados de las hipótesis confirman que el alto compromiso con la equidad de género de Scotiabank Perú, como parte de su responsabilidad social corporativa se relaciona de manera directa con su nivel de competitividad.

Se ha comprobado que existe relación significativa entre la responsabilidad social bancaria y la competitividad en el Banco sujeto de estudio, según la prueba correlación de Spearman, cuyo valor del coeficiente Rho es 0,930, que es significativa. Los hallazgos son similares al informe de Guerra, Medina y Ríos (2016). Desde la perspectiva de la responsabilidad social empresarial, la industria bancaria peruana tiene un mayor grado de integración con los grupos de interés, enfocándose en sus procesos, políticas y estrategias para lograr el respeto al consumidor. Excelente servicio al cliente y ventas éticas. En cuanto a grupos de interés, accionistas y proveedores, se puede considerar que el departamento ha realizado acciones para establecer relaciones con la competencia, establecer principios éticos y comprometerse con el buen gobierno corporativo. Por el lado de los proveedores, existe un proceso estricto y transparente para evaluarlos y seleccionarlos. Los resultados hipotéticos confirman que al establecer buenas relaciones con los grupos de interés mejora el nivel de competitividad.

\section{CONCLUSIONES Y RECOMENDACIONES}

Se ha comprobado que la responsabilidad social bancaria influye significativamente en la competitividad en el Banco sujeto de estudio, sede Tacna, año 2019. La responsabilidad social bancaria tiene un nivel de influencia del $72 \%$ sobre la variable competitividad. El nivel de competitividad en el Banco fue regular.

Así se recomienda a los directivos del Banco, sede Tacna disponer que se desarrolle una propuesta de Gestión de la Responsabilidad Social Bancaria, con enfoque de innovación, y priorizando a los grupos de interés, para elevar la competitividad en el Banco. El tipo de enfoque de innovación que ayudará a la propuesta es el de la OCDE - Organización para la Cooperación y el Desarrollo Económico (2005), específicamente en innovación organizacional. El Jefe del Área de Gestión de la Responsabilidad Social debe realizar un seguimiento al aspecto ético, para elevar la competitividad en el Banco. El Modelo para la Evaluación de Programas de Ética Organizacional ayudará al seguimiento de la dimensión. Se debe desarrollar un sistema de gestión de la dimensión colaboradores para optimizar la competitividad creando un Área de Responsabilidad Social o Comité de Responsabilidad Social, donde se establezcan políticas efectivas de relaciones con los colaboradores, programas de desarrollo profesional de Seguridad y Salud Ocupacional para los trabajadores. Se debe desarrollar un seguimiento a la dimensión ambiental para mejorar competitividad, a través de los siguientes 
indicadores: Instrumento de gestión ambiental, Implementación de políticas ambientales; estrategias de comunicación ambiental.

\section{REFERENCIAS}

Hidalgo, O. (2018). Influencia de la responsabilidad social empresarial en la imagen institucional de las entidades Financieras de Tacna, año 2017. (tesis de doctorado). Universidad Privada de Tacna.

Aranda, A. (2015). La responsabilidad social como factor estratégico en el sector de las Cajas de Ahorro. (Tesis Doctoral). Universidad de Jaén - España

Aránzazu, J. (2019). Responsabilidad social corporativa ADGG072PO. Málaga- España: Editorial IC

BBVA Continental. (2015a). Política medioambiental del Grupo BBVA. Recuperado de http://bancaresponsable.com/informe-anual-2009/pdf/Politica-Medioambiental-del- GrupoBBVA.pdf

Beltrán, D.; Quintana, E., e Hidalgo, K. (2016). Responsabilidad social empresarial en el sector financiero. análisis de una empresa local a través del benchmarking de 3 empresas extranjeras en buenas prácticas en la gestión de equidad de género, durante el periodo 2014-2016. (Trabajo de suficiencia profesional).Universidad Peruana de Ciencias Aplicadas - Lima.

Bocanegra, H. (2010). El Componente comunicacional en los programas de responsabilidad social empresarial en las entidades financieras peruanas: Caso del Banco de la Nación. (Tesis de grado). Universidad Nacional Mayor de San Marcos. Lima.

Camejo, A. (2018). La emergentge ética y responsabilidad social en las relaciones laborales en el siglo XXI. Bogotá-Colombia. Editorial del Politécnico Grancolombiano, Asociación de Editoriales Universitarias de Colmbia - ASEUC

Canto, G.; Guerra, R.; Medina, L. y Ríos, A. (2016). Diagnóstico y propuestas de mejora para una banca socialmente responsable, en el Perú. (Tesis de maestría). Pontificia Universidad Católica del Perú.

Cardona, M.; Rendón, J. (2013). Responsabilidad Social Empresarial: Teorías, índices, estándares y certificaciones. Universidad del Valle/Vol. 29 Nro. 50/julio-diciembre de 2013. Recuperado de https://www.redalyc.org/html/2250/225029797009/

Cea, R. (2010). La responsabilidad social corporativa en las entidades bancarias de la Unión Europea. Análisis empírico y propuesta de modelo normalizado (Tesis Doctoral). Universidad Autónoma de Madrid.

Corredor, B. et al (2016). El Estado de la gestión con enfoque en responsabilidad social empresarial en los cinco principales bancos en Colombia. (Tesis de Maestría). Pontificia Universidad Católica del Perú.

Emil, V. (2011). Competitividad de la empresa y los distritos industriales. España: Editorial Académica. Gil, G. (2018). Responsabilidad social corporativa: revisión crítica de una noción empresarial. Madrid España. Editorial Centro de Investigaciones sociológicas

Navarro, F. (2012). Responsabilidad Social Corporativa: Teoría y práctica. Madrid: Editorial ESIC

Rosero (2015). Relación entre desempeño financiero y responsabilidad social empresarial: un análisis en la Banca europea. (Tesis Doctoral). Universidad de Valladolid - España.

Truño, J. (2016). La responsabilidad social corporativa, una aproximación en el sector bancario. (Tesis de doctorado). Universitat Autónoma de Barcelona. España.

Uribe, M. (2012). Competitividad empresarial. Editorial. EAE.

Vidal, I., y Morrós, J. (2015). Responsabilidad Social Sostenibilidad GRI e ISO 26000. Madrid, España: Fundación Confemetal

Velarde, J. (2019). La responsabilidad social empresarial y su influencia en el posicionamiento de las entidades bancarias en Tacna, 2018. (tesis de doctorado). Universidad Nacional Jorge Basadre Grohmann- Tacna.

Wong, C. (2015). Responsabilidad social y su aplicación en los créditos bancarios (Tesis de Grado). Universidad de Guayaquil - Ecuado 